\title{
Assessment of Spatial Accessibility to Residential Care Facilities in 2020 in Guangzhou by Small-Scale Residential Community Data
}

\author{
Danni Wang ${ }^{1,2}$, Changjian Qiao ${ }^{3}$, Sijie Liu ${ }^{4}$, Chongyang Wang ${ }^{2,5, * \mathbb{C}}$, Ji Yang ${ }^{2,5}$, Yong $\mathrm{Li}^{2,5}$ and \\ Peng Huang ${ }^{6}$ \\ 1 Department of Resources and the Urban Planning, Xin Hua College of Sun Yat-Sen University, \\ Guangzhou 510520, China; wangdanni@xhsysu.edu.cn \\ 2 Southern Marine Science and Engineering Guangdong Laboratory (Guangzhou), Guangzhou 511458, China; \\ yangji@gdas.ac.cn (J.Y.); liyong@gdas.ac.cn (Y.L.) \\ 3 College of Resources and Environment, Academician Workstation for Urban-Rural Spatial Data Mining, \\ Henan University of Economics and Law, Zhengzhou 450046, China; cjqiao@whu.edu.cn \\ 4 Land and Resources Technology Center of Guangdong Province, Guangzhou 510075, China; \\ liusijie0808@gmail.com \\ 5 Key Lab of Guangdong for Utilization of Remote Sensing and Geographical Information System, \\ Guangdong Open Laboratory of Geospatial Information Technology and Application, Guangzhou Institute \\ of Geography, Guangzhou 510070, China \\ 6 Shenzhen Municipal Planning and Natural Resources Bureau-Bao'an Management Bureau, \\ Shenzhen 518101, China; wgldwj@gmail.com \\ * Correspondence: wangchongyang@gdas.ac.cn; Tel.: +86-188-0208-0904
}

Received: 27 February 2020; Accepted: 12 April 2020; Published: 15 April 2020

check for updates

\begin{abstract}
Population aging has increasingly challenged socio-economic development worldwide, highlighting the significance of relevant research such as accessibility to residential care facilities (RCFs). However, a number of previous studies are carried out only on street (town)-to-district scales, which could cause errors of the accessibility to RCFs for a family. In order to improve the resolution to individual families, we measure and compare the accessibilities to RCFs based on 3494 residential communities and 169 streets of Guangzhou in 2020 through the two-step floating catchment area (2SFCA) method. It was found that the distributions of the elderly and the service-dense blobs of the RCFs show patterns of a three-level spatial distribution, with a characteristic clustering at the center with peripheral dispersion. The resultant accessibility to RCFs in Guangzhou, ranging from 2.5 to 3.45 , is generally consistent with the studies focusing on street scales. However, the maximum difference in the accessibility of two residential communities on the same street ranges from less than 0.02 to 0.94 in Guangzhou, indicating large variations. Although the relative errors of the accessibility results based on bi-scale data are relatively low, the cumulative errors can be high, e.g., over $25 \%$ in many streets of large cities. Consequently, hundreds of elderly persons per street can be adversely affected by those errors, with six streets over 1000. Therefore, this study focusing on the smaller-scale residential community data may provide more accurate reference to individual households. For the spatial allocation and optimal layout of Guangzhou and similar cities with population aging, we suggest maximizing RCFs in metropolises by taking full advantage of existing residential care facilities with necessary restructuring, improvements, and expansions on service capability. While for less connected cities, we encourage building new RCFs in situ.
\end{abstract}

Keywords: spatial accessibility; residential care facilities; residential communities; population forecast; Guangzhou 


\section{Introduction}

Population aging has become an increasingly prominent phenomenon in the world [1]. It brings significant challenges and impacts on many fronts of sustainable development [2,3]. Since the 1990s, many cities of countries have entered an aging society, and the number is continuously increasing. For the third-largest city in China, Guangzhou, reports in 2017 and 2018 from the government showed that the elderly population (aged 60 or older) size is growing at a rate of $5 \%$ per year [4,5], with predictions of reaching 1.85 million by 2020 , accounting for almost $20 \%$ of the total population.

The spatial accessibility to public services, such as residential care facilities, hospitals, health services, markets, parks, sport facilities, schools, and banks, is a critical issue in the field of public health, reasonable urban planning and sustainable development [6,7]. It has a significant impact on social equity and justice, especially for an aging society [8]. Therefore, it could find that a number of studies have focused on measuring spatial accessibility to public services worldwide, including cities and other developing regions [9-11].

\section{Literature Review}

A variety of methods are commonly applied to measuring spatial accessibility, such as interviews, supply-to-demand ratio, distance to the closest facility, kernel density, gravity model and floating catchment area method [2,12-18].

Based on the Gaussian kernel density method, Guagliardo. (2004) gave an example of identifying the areas with a low ratio of primary care physicians to children in Washington [14]. Cheng et al. (2011) used in-depth semi-structured interviews to assess the well-being of elderly residents in residential care facilities (RCFs), and further analyzed the influence of its environment on the elderly activities and health [2]. Bauer et al. (2018) measured spatial general practitioners accessibility by a floating catchment area method and found that general practitioners' accessibility for $25.8 \%$ of the population in England was very low, while the mostly high general practitioners accessibility areas represented urban areas [12]. Yu et al. (2018) used a two-step floating catchment area to compare the spatial accessibility of health services within the current and planned network systems in Shenzhen [19]. It was found that the impacts on spatial accessibility of health services had spatial variations, and the most positively and negatively affected places were mainly in the center zone. Through an improved two-step floating catchment area method, Tao et al. (2014) analyzed the spatial accessibility to residential care resources in Beijing [20]. The results showed that the spatial accessibility was higher in the western, northern and northeastern parts of the city than the southern part. Besides, Tao et al. (2018) also measured healthcare accessibility in Shenzhen and found that disparity in transit-mode accessibility is the main reason for the imbalance of healthcare accessibility [17].

It should be noted that those methods of measuring spatial accessibility had both advantages and disadvantages when they were applied to different conditions $[3,13,20,21]$. In contrast, the floating catchment area may be the most popular and commonly-used method because it takes into account maximum influence factors of spatial accessibility, for instance, the size of supply-side and demand-side, distance and the distance limits $[13,14,17,21-23]$.

In addition, many previous studies about spatial accessibility including the assessment of the accessibility to RCFs were mainly based on the street (town)-to-district scale $[3,12,14,17,18,20,21,24-28]$, especially in cities of China. Streets (towns) are one of the administrative divisions smaller than a district in a city and the minimum statistical unit in Census in China. However, these studies might have in general overlooked the sub-structural differences in a street or town, which could cause errors of accessibility to RCFs for an individual family. For example, in the central city of Guangzhou, the maximum distances of two different families in the same street (town) are about $7.5 \mathrm{~km}$ in the Haizhu District, $4.8 \mathrm{~km}$ in the Tianhe District, $3.95 \mathrm{~km}$ in the Yuexiu District and $3.1 \mathrm{~km}$ in the Liwan District. Those distances can be larger for the main and surrounding cities. The interior difference indicates the possibility that one family has to spend significantly more time (20 min to one hour) to 
reach $\mathrm{RCFs}$ than another family in the same street. Obviously, specific information for a family to access RCFs based only on street scale data is absent.

This study intends to measure and compare the difference of spatial accessibilities to RCFs on the residential communities and street scales, and provide more detail and precise information of the accessibility for families in Guangzhou. To achieve the goal, the spatial distribution characteristics of the elderly population in 2020 and RCFs in Guangzhou were analyzed firstly. Then, the accessibilities to RCFs based on residential community data and streets of Guangzhou were calculated and compared by the most commonly-used two-step floating catchment area (2SFCA) method. Finally, this study provides suggestions for the development of RCFs and urban planning in Guangzhou.

\section{Dataset and Method}

\subsection{Study Area}

Located between longitudes $112.95^{\circ}-114.05^{\circ}$ E and latitudes $22.43^{\circ}-23.93^{\circ} \mathrm{N}$ (Figure 1), Guangzhou is the political, economic, scientific, educational and cultural center of Guangdong Province, China (http://www.gz.gov.cn/zlgz/index.html). Guangzhou includes 134 streets and 35 towns under the 11 districts (Table 1).

Table 1. Administrative division, residential community data, residential care facilities (RCFs) and its service intensity-beds, and the elderly population of Guangzhou.

\begin{tabular}{|c|c|c|c|c|c|}
\hline Guangzhou & District & $\begin{array}{l}\text { Streets } \\
\text { (Towns) }\end{array}$ & $\begin{array}{l}\text { Residential } \\
\text { Communities }\end{array}$ & $\begin{array}{c}\text { RCFs (beds < } \\
100,100-300 \\
>300)\end{array}$ & $\begin{array}{l}\text { The Elderly } \\
\text { Population }\end{array}$ \\
\hline \multirow{4}{*}{ The central city } & Yuexiu & 18 & 512 & $19(10,8,1)$ & 290,082 \\
\hline & Tianhe & 21 & 573 & $12(9,1,2)$ & 152,883 \\
\hline & Haizhu & 18 & 618 & $26(11,11,4)$ & 297,629 \\
\hline & Liwan & 22 & 382 & $30(11,14,5)$ & 205,478 \\
\hline \multirow{4}{*}{ The main city } & Baiyun & 22 & 421 & $31(4,16,11)$ & 224,552 \\
\hline & Huangpu & 14 & 144 & $10(2,2,6)$ & 79,940 \\
\hline & Panyu & 16 & 562 & $17(4,9,4)$ & 155,355 \\
\hline & Nansha & 9 & 39 & $10(5,4,1)$ & 76,060 \\
\hline \multirow{3}{*}{$\begin{array}{l}\text { The } \\
\text { surrounding } \\
\text { city }\end{array}$} & Huadu & 10 & 115 & $13(6,5,2)$ & 127,853 \\
\hline & Zengcheng & 11 & 96 & $11(3,7,1)$ & 147,673 \\
\hline & Conghua & 8 & 32 & $10(9,1,0)$ & 92,495 \\
\hline Total & 11 & 169 & 3494 & 189 & $1,850,000$ \\
\hline
\end{tabular}

Note: The elderly population was forecasted for 2020 using a natural growth method (Tao et al., 2015) based on 2010, 2017 and 2018 data. 


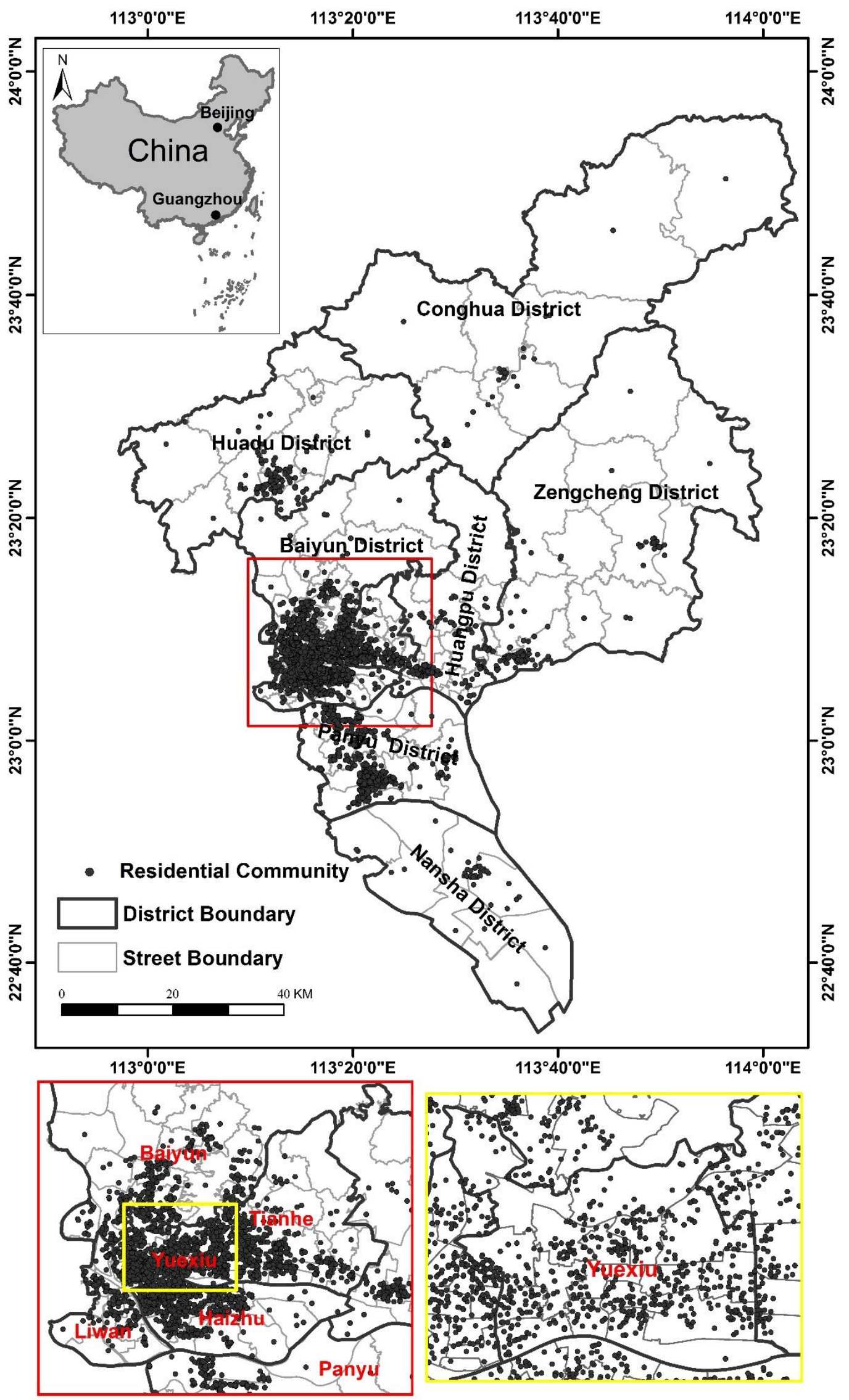

Figure 1. Spatial distributions of 3494 residential communities and 169 streets in Guangzhou. 


\subsection{Data and Preprocessing}

In order to assess the spatial accessibility to RCFs on different scales, this study collected residential communities, streets data, RCFs, and road network of Guangzhou in 2019, and forecasted the elderly population in 2020.

Based on the web crawler technique, 3478 pieces of residential location data in 153 streets (towns) of Guangzhou were obtained thanks to open access of data from the "Lianjia" website (https://gz.lianjia.com/), labeled as the residential community data in the study. However, the residential community data in the other four streets and 12 towns mainly located in the suburban areas were not obtained, due to the low urbanization degree and their resemblance to villages, with little validated data on residential location. Therefore, the geometric centers of these streets and towns were used to replace its residential communities. There are a total of 3494 residential communities in the study area (Table 1 and Figure 1).

Residential care facility data was collected from the website of the Guangzhou Civil Affairs Bureau (http://www.gzmz.gov.cn/gzsmzj/lhyljgxx/pglh_yljgxx.shtml), including its name, type, address and number of beds, which are all made open access. By April 2019, there were 189 RCFs in Guangzhou (Table 1). We used the geocoding service of the Baidu map to acquire the geographic locations of the RCFs and its spatial distribution and service intensity (bed numbers) were shown in Figure 2.

Road network data was provided by the Land and Resources Technology Center of Guangdong Province. The speed limits of different types of roads (Table 2, Figure 3) in Guangzhou were obtained mainly via the Urban Road Engineering Design Specification (CJJ37-2012) and the Highway Engineering Technical Standard (JTG B01-2014), which could be regarded as a universal standard in many cities in China, such as Beijing, Guangzhou and Shenzhen.

Table 2. Speed settings of different road types in Guangzhou.

\begin{tabular}{cccccc}
\hline Type & Highway & State Road & Provincial Road & County Road & Township Road \\
\hline Speed $(\mathrm{km} / \mathrm{h})$ & 80 & 60 & 40 & 30 & 20 \\
\hline
\end{tabular}

According to the sixth national census in 2010, the elderly population was 1.23 million in Guangzhou and showed a rapid growth trend [29]. By 2017 and 2018, the government reported that the elderly population had grown to 1.61 million and 1.69 million [4,5].

Based on the population data in 2010, 2017 and 2018, this study forecasted the elderly population in 2020 through a natural growth method [8]. It was found that the elderly population will reach about 1.85 million in 2020 in Guangzhou (Table 1). Among all the districts of Guangzhou, the elderly population in Yuexiu, Haizhu, and Liwan Districts are more than 200 thousand, and in the other five districts (Tianhe, Baiyu, Panyu, Zengchen and Huadou Districts), the elderly population are over 100 thousand (Table 1). When calculating the spatial accessibility, the number of elderly people of each district is assumed to be evenly distributed across the residential communities. 


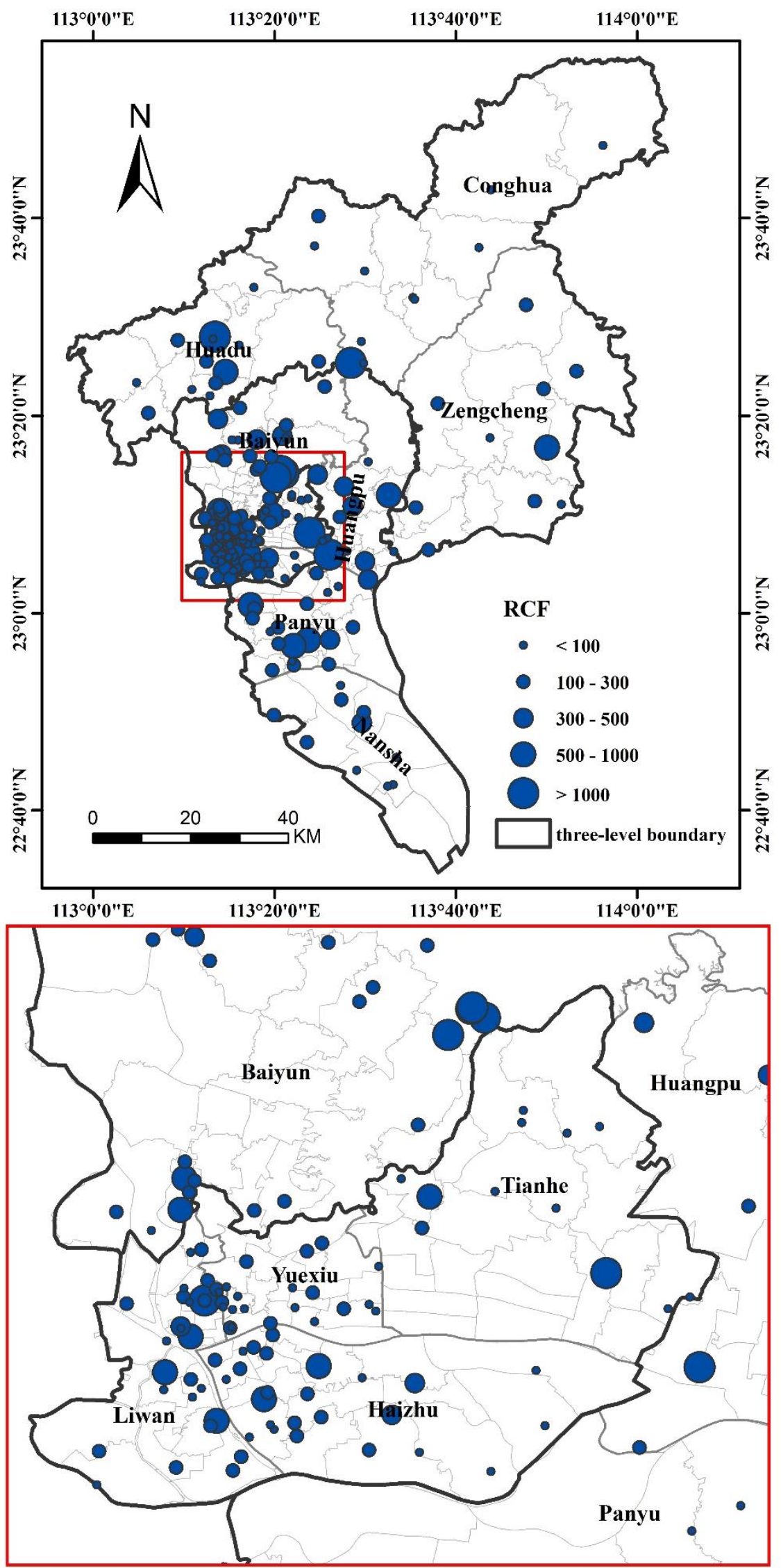

Figure 2. Spatial distributions of 189 RCFs and its service intensity (beds) in Guangzhou. 

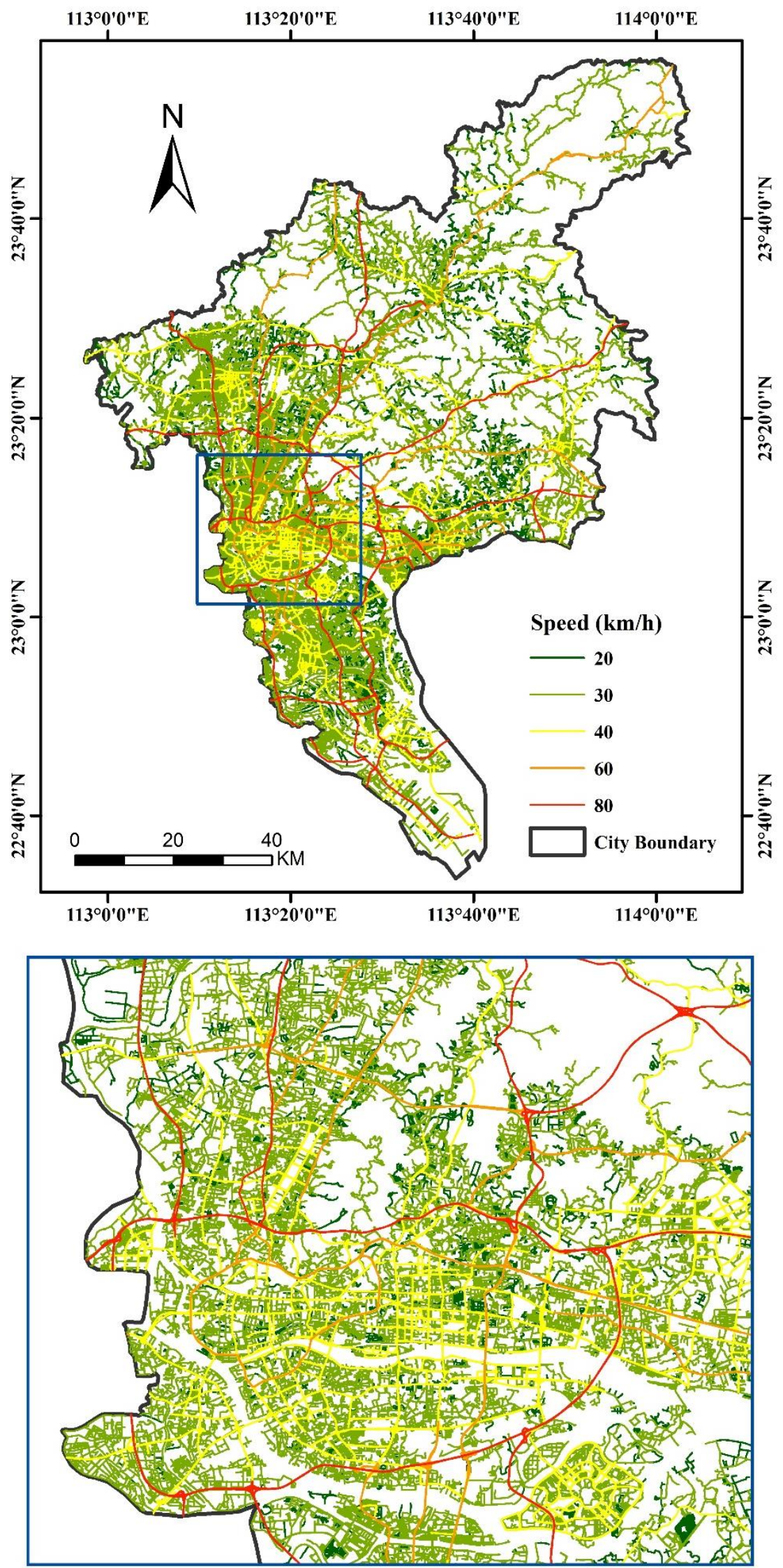

Figure 3. Road network and its speed attributes in Guangzhou. 


\subsection{Method of Measuring Spatial Accessibility}

The 2SFCA method was proposed by Radke and Mu (2000) based on the early moving search method in 2000 [15]. The method first sets a threshold value using the travel limit distance or time as the search radius, and the moving search is performed twice based on the supply location and the demand ground, respectively. The number of resources or facilities accessible to the residents within the threshold is calculated. The higher the result is, the better the accessibility to the resources or facilities [30]. The 2SFCA method is not limited to administrative boundaries, overcoming the shortcomings of traditional methods. In recent years, more and more scholars have used the 2SFCA method to study the accessibility of public service facilities [22,23,27]. The method uses the micro-scale, such as the residential or street population densities to study the spatial features of the regional population. At the same time, the spatial distribution of the nursing homes and the number of beds are included in the accessibility evaluation, which could be used to analyze the interaction between the residents and the nursing homes more reasonably, an advantage of the 2SFCA method.

In the study, the 2SFCAmethod was selected to measure the accessibilities to RCFs based on the residential communities and streets data in Guangzhou. The basic idea of the method is to set a search radius by travel limit distance or time and calculate the moving search results based on the supply location and demand ground thereafter. When measuring and assessing the spatial accessibilities, both the spatial distribution of RCFs and its service intensity are taken into account by the 2SFCA method, which could acquire a more reasonable result $[13,23,24,27,31]$.

In the first step, the supply-to-demand ratio $\left(R_{j}\right)$ for each $\operatorname{RCF}(j)$ is calculated based on the search result of the resident community $(k)$ within the distance threshold $\left(d_{0}\right.$, service radius). The RCFs are the supply nodes, and the resident communities are the demand nodes.

$$
R_{j}=\frac{S_{j}}{\sum_{k \in\left\{d_{k j} \leq d_{0}\right\}} P_{k}}
$$

where $P_{k}$ is the amount of the elderly in resident community $(k), S_{j}$ is the amount of the total supply (such as the beds) for RCF $(j), d_{k j}$ is the distance between resident community $(k)$ and RCF $(j)$.

In the second step, we add the supply-to-demand ratios $\left(R_{j}\right)$ of all RCFs located within the distance threshold $\left(d_{0}\right)$ of the resident community $(i)$. The sum of supply-to-demand ratios $\left(R_{j}\right)$ for each resident community (i) is its spatial accessibility score (Equation (2)).

$$
A_{i}^{F}=\sum_{j \in\left\{d_{i j} \leq d_{0}\right\}} R_{j}
$$

where $A_{i}{ }^{F}$ is the spatial accessibility score of the residential community (i) to all RCFs located within the distance threshold $\left(d_{0}\right)$. The higher the score is, the better the accessibility to the residential community. $R_{j}$ is the supply-to-demand ratio for each RCF $(j)$ within the search area $\left(d_{i j} \leq d_{0}\right)$ of the residential community (i).

The first step above determines the workload of the RCFs, that is, the supply-demand ratio of each RCF within the service radius. The second step calculates the accessibility of each residential community, and retrieves all RCFs that can provide services for the residential community. The supply-demand ratio obtained in the first step is summed up, and the sum is the accessibility value that represents the number of beds per 100 elderly (BP100E). This method takes into account the relationship between supply and demand in an area, and the accessibility value calculated by each residential community is different. In addition, different scales of RCFs have different service radii. Referring to the existing research, the service radii of large, medium and small scales of RCFs were set as 1.5, 1.0, $0.5 \mathrm{~h}$ respectively, which were used to calculate the value of BP100E in each residential community. 


\section{Results and Discussions}

\subsection{The Spatial Distribution Characteristics of RCFs in Guangzhou}

Based on the RCFs data, it was found that the spatial distribution of RCFs in Guangzhou had high variation (Figure 2). Respectively, 87, 68 and 34 RCFs were in the central, main and surrounding cities by 2019. For the distribution of RCFs, we find the central city has a significant clustering characteristic, with less in main cities and the least in the surrounding cities (Table 1). In addition, a total of 115 streets include at least one residential care facility, which illustrated that the distribution of RCFs was relatively balanced in Guangzhou.

Furthermore, the 189 RCFs in Guangzhou are labeled as three types: small, medium and large, based on the service intensity (Figure 2, Table 1). There were 74 small RCFs (Beds less than 100), 37 large RCFs (Beds more than 300) and 78 medium RCFs (Beds in the middle). It was also found that there were more small (55\%) and medium (44\%) RCFs in the central city than that in main and surrounding cities, mainly due to the presence of a large elderly population with limited space in these areas. In the main city, however, there were more medium (40\%) and large (59\%) RCFs. It should mainly attribute to the characteristics of abundant space in the main city and the urban function of transferring the pressure of the central city (Figure 2).

The results from kernel analysis indicate that RCFs are clustered in urban centers and reaching out to the surrounding areas (Figure 4). Within the central urban area, the RCFs are mainly distributed in Yuexiu and Liwan Districts, the western part of the Haizhu District, and the northwest of the Tianhe District, which forms a southwest-northeast axis connected with the southwestern part of Baiyun District. Near the main kernel, the other hotspots appear in the middle and south of Huangpu District, the northwest and south of Panyu District, the south-central part of Huadu District, the eastern part of Zengcheng District, and the junctional region between Huadu, Baiyun and Conghua Districts (Figure 4). The spatial distribution of RCFs shows the spatial pattern of the cluster development in the main core and the balanced development in many high-value areas in Guangzhou. 

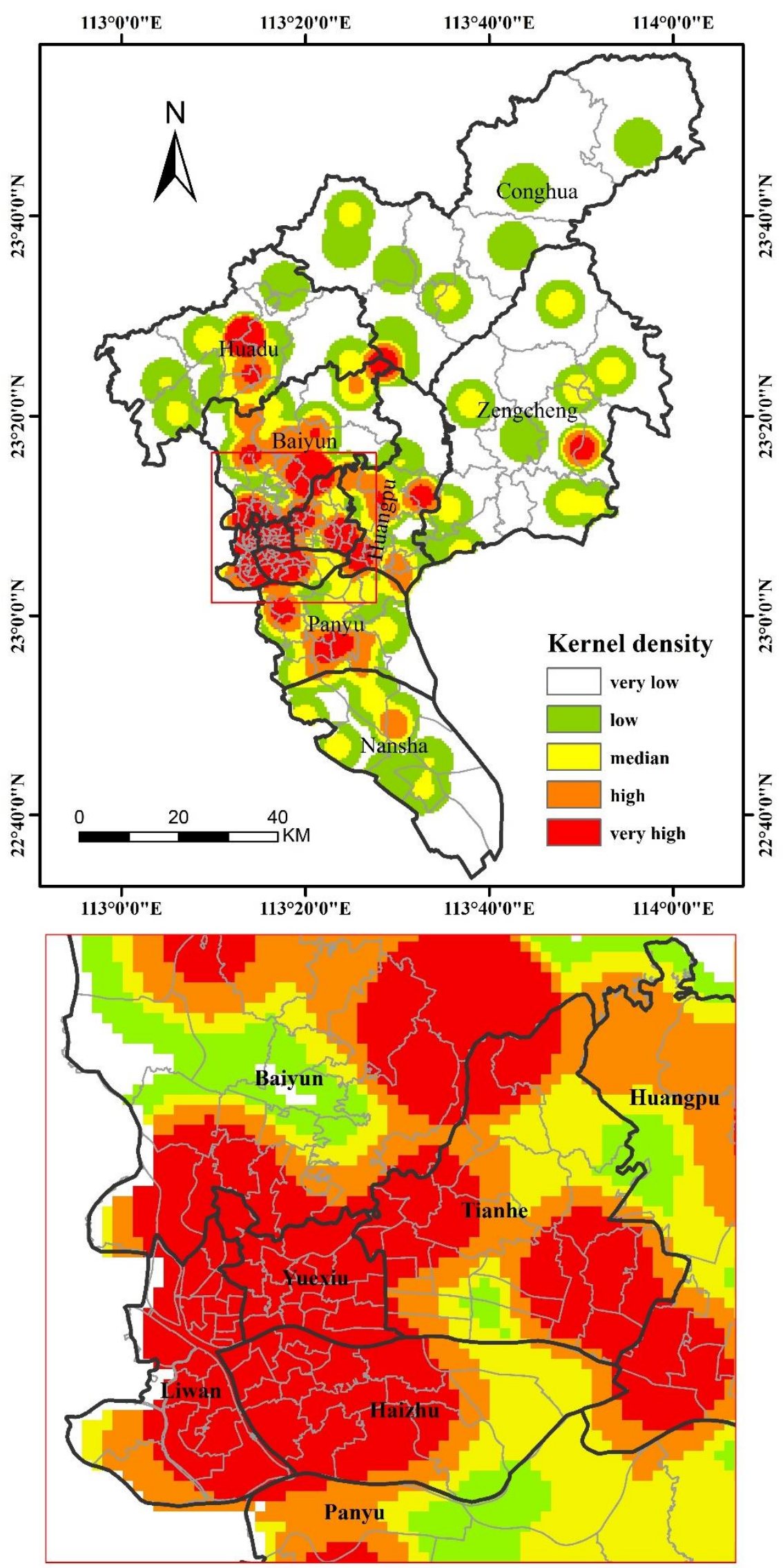

Figure 4. Kernel analysis of RCFs in Guangzhou. 


\subsection{The Spatial Variations of the Elderly in 2020}

In the forecasted elderly population density map in 2020 in Guangzhou, we found a high-medium-low tri-level spatial distribution pattern from the central city to the periphery (Figure 5). In the central, main and surrounding cities of Guangzhou, the elderly accounts for $51.14 \%, 28.96 \%$ and $19.9 \%$ of the population, respectively. The average density of the elderly population is 2931 (persons $/ \mathrm{km}^{2}$ ) in each street. In particular, 36 streets have more than 5000, such as Yuexiu district, northeast of Liwan district and northwest of Haizhu district in the central city. The street with the highest density is Dadong Street in Yuexiu District, the core of the central city, up to 19,903 (persons $/ \mathrm{km}^{2}$ ). While Lvtian town, Conghua District, located on the edge of the city, has the lowest density (11 persons $/ \mathrm{km}^{2}$ ). According to the distributions of the RCFs and the elderly in Guangzhou, it was found that the heat map of the RCFs follows the density map of the elderly population, where the service-dense blobs overlap the ones of the elderly population.

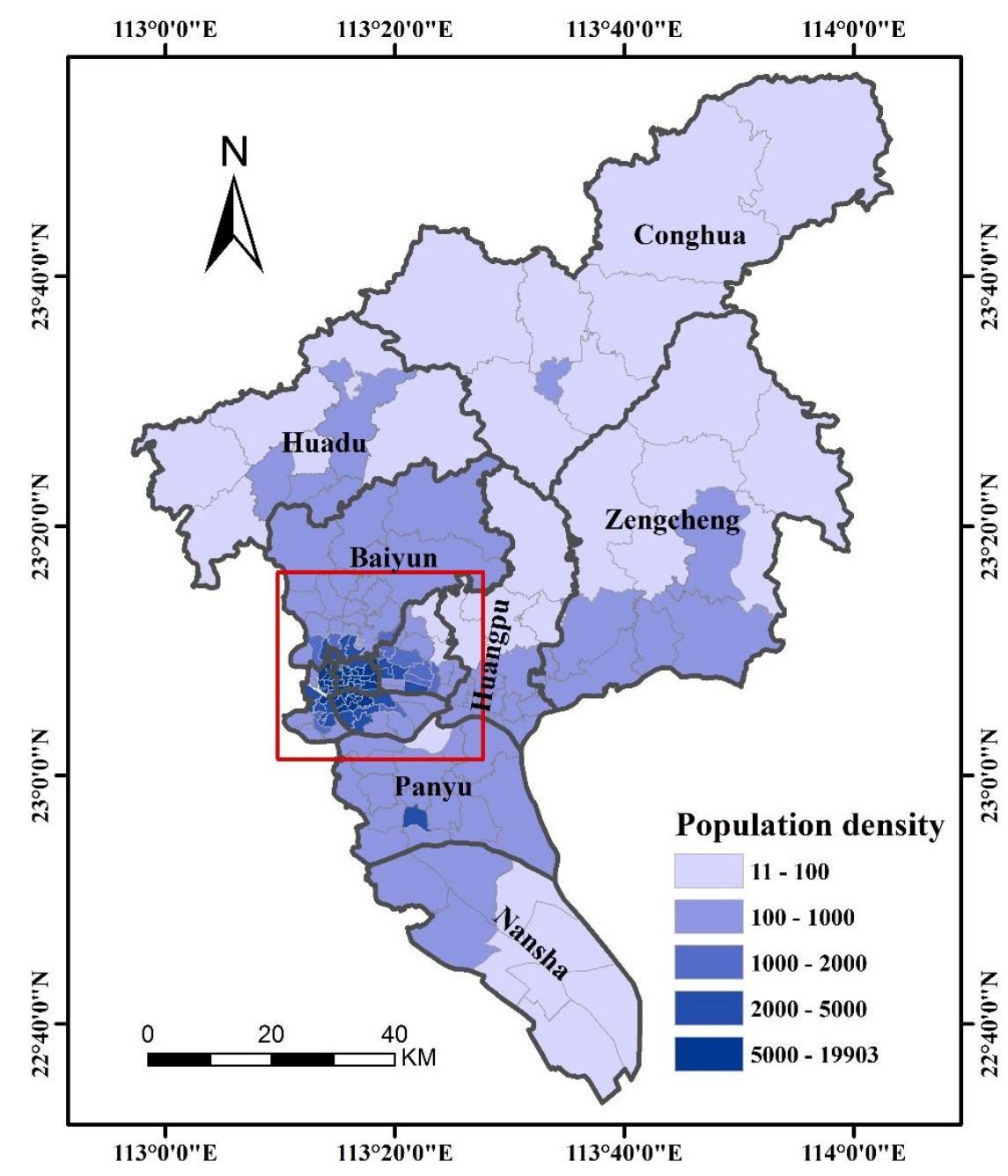

Figure 5. Cont. 


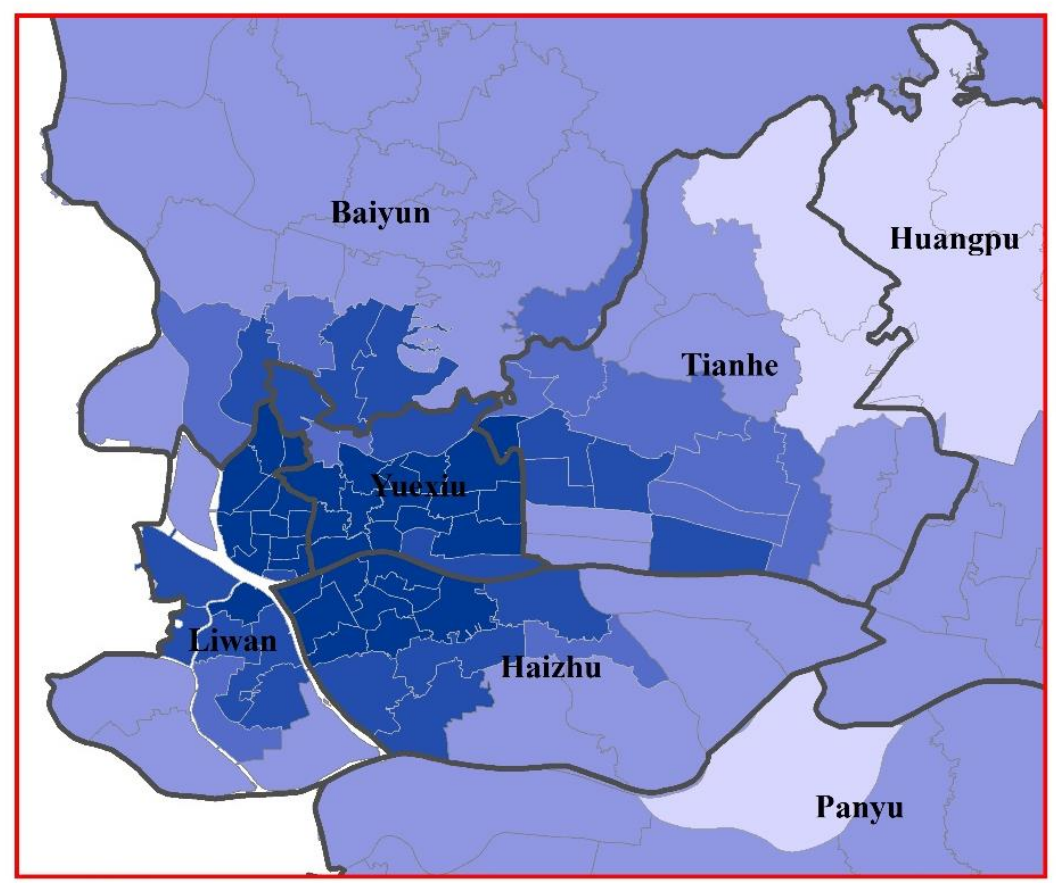

Figure 5. The spatial variation of the elderly population at the streets level in 2020.

\subsection{The Accessibilities to RCFs in Guangzhou on Residential Community and Street Scale}

In this study, the 2SFCA method is applied for the measurement and analysis of the spatial accessibilities to RCFs, at both residential community and street scales. The spatial accessibility to RCFs was represented as the number of beds per 100 elderly (BP100E). The higher value of BP100E is, the higher accessibility to RCFs is.

Figure 6 showed the calculated results of the BP100E on the street scale and the residential community scale. Note that the BP100E shows a pattern of "central city $>$ main area $>$ surrounding area". In the central area, the accessibility of residential communities to RCFs has a decreasing trend from east to west and in the main area from north to south. The accessibility decreases with the distance to the central area, which also holds for main cities. From Figure 6, a certain degree of spatial clustering and heterogeneity is evident from the BP100E on the street scale. It reflects the spatial clustering of residential areas with similar service levels, which is consistent with the street-scale results. Heterogeneity reflects fluctuating service levels across certain areas, with outliers. It is speculated that the RCFs planning had ignored the absolute number of settlements and instead incorrectly used the geometric center as reference. 

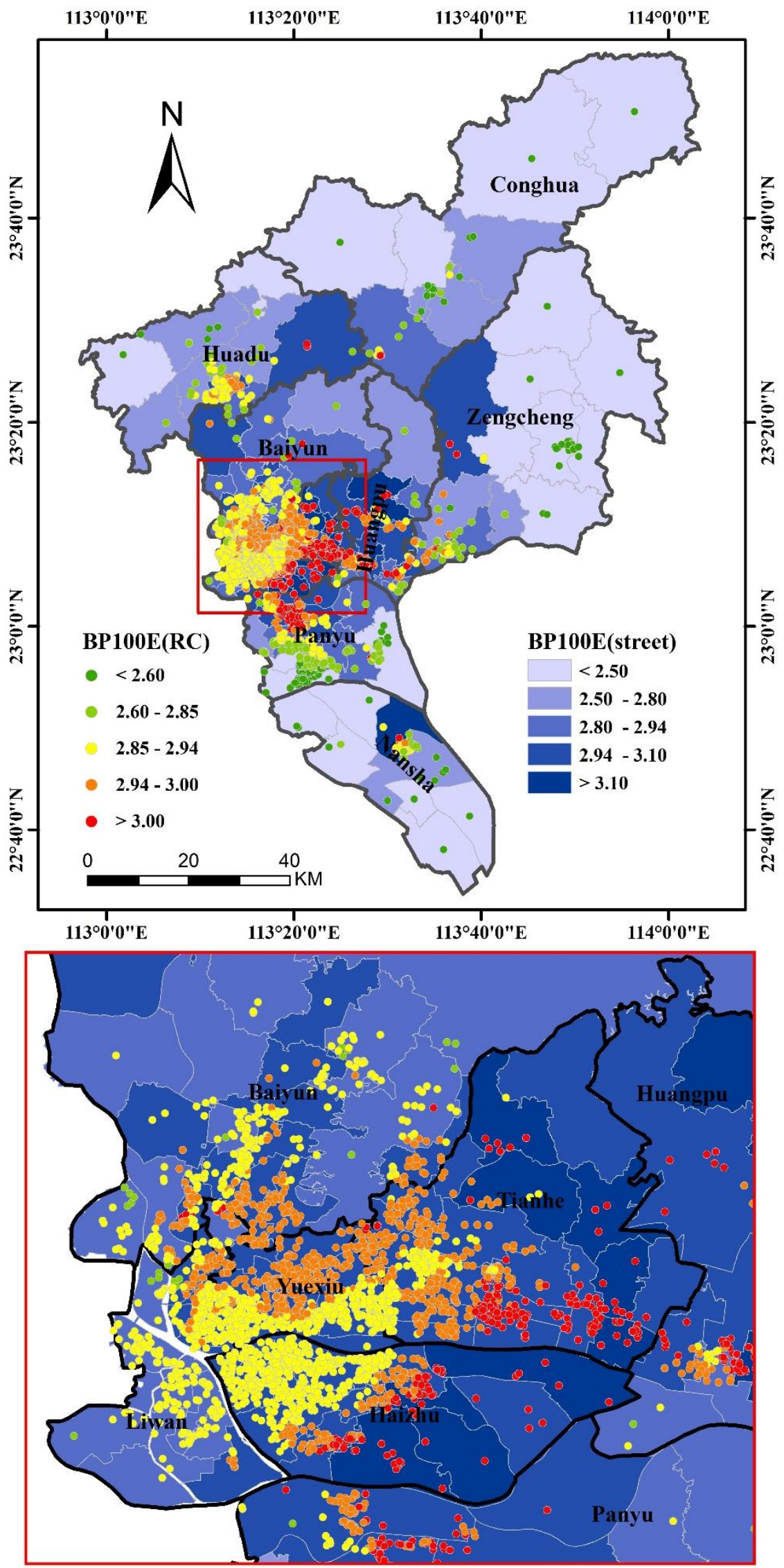

Figure 6. Spatial accessibilities to RCFs based on street scale and residential community scale in 2020 in Guangzhou. 
Table 3 shows the statistics of the BP100E in each district. Among the top 50 streets, 30 belongs to the central area. The averaged BP100E is higher in Tianhe (3.05), Haizhu (3.01) and Yuexiu (2.97) districts and with both Tianhe and Haizhu having BP100E > 3.0 on the street scale. Liwan has a lower value of 2.8 but is still high in Guangzhou. The reason is that the rate of the aged is low in the Tianhe District and high in and around Yuexiu District. For the main cities, there are 18 streets in the top 50 (rightmost column) and the average value of BP100E is 2.93 and 3.00 in Baiyun and Huangpu District, respectively. In Huangpu District, a fraction of $43.91 \%$ of the aged population are covered by BP100E $>3.0$, which is mainly distributed in the vicinity of the central area. However, $41.47 \%$ are living in the area with $\mathrm{BP} 100 \mathrm{E}<2.9$, mainly from the surrounding areas. Only two streets belong to the top 50 in the surrounding areas, and the proportion of elderly people served with the BP100E $<2.9$ is $61.65 \%, 91.82 \%$, and 100\% in Huadu, Zengcheng and Conghua District, respectively, indicating low accessibility of RCFs in the surrounding areas.

Table 3. Characteristic statistics of the number of beds per 100 elderly (BP100E) in each district.

\begin{tabular}{ccccccc}
\hline \multirow{2}{*}{ District } & BP100E & \multicolumn{3}{c}{ Streets, Aging Population (\%) } & \multirow{2}{*}{$\begin{array}{c}\text { Streets Count of } \\
\text { Top 50 }\end{array}$} \\
\cline { 3 - 6 } & & \multicolumn{2}{c}{ BP100E $<$ 2.9 } & \multicolumn{2}{c}{ BP100E $>$ 3.0 } & 5 \\
Yuexiu & $2.88-3.01$ & 1 & 3.80 & 1 & 1.25 & 15 \\
Tianhe & $2.95-3.29$ & 0 & 0 & 11 & 48.00 & 7 \\
Haizhu & $2.95-3.14$ & 0 & 0 & 6 & 14.29 & 3 \\
Liwan & $2.88-3.00$ & 4 & 4.46 & 0 & 0 & 6 \\
\hline Baiyun & $2.77-3.03$ & 7 & 36.99 & 4 & 23.72 & 8 \\
Huangpu & $2.76-3.32$ & 5 & 41.17 & 7 & 43.91 & 3 \\
Panyu & $2.01-3.09$ & 12 & 75.61 & 2 & 7.06 & 1 \\
Nansha & $1.05-3.46$ & 8 & 89.73 & 1 & 10.27 & 1 \\
\hline Huadu & $2.00-3.08$ & 7 & 61.65 & 1 & 15.06 & 1 \\
Zengcheng & $1.41-3.05$ & 10 & 91.82 & 1 & 8.18 & 0 \\
Conghua & $0.51-2.86$ & 8 & 100 & 0 & 0 & \\
\hline
\end{tabular}

In order to analyze the spatial characteristics of clustering and heterogeneity about BP100E at the residential community scale, we used "Global Moran's I" to analyze its spatial autocorrelation first. The results were shown in Table 4.

Table 4. Global Moran's I Summary.

\begin{tabular}{ccccc}
\hline Moran's Index & Expected Index & Variance & z-Score & $p$-Value \\
\hline 0.52 & 0.00 & 0.00 & 266.20 & 0.00 \\
\hline
\end{tabular}

Given the z-score of 266.20 and BP100E of 0, the likelihood for chance coincidence is less than $1 \%$. Therefore, we used the Local Indicators of Spatial Association (LISA) to analyze the spatial characteristics of the BP100E at the residential community scale. The results of the cluster and outlier analysis show statistically significant high-value and low-value clusters.

In Figure 7, the distribution of the BP100E on the residential community-scale is generally consistent with the one on the street scale. The high-value spots (green dots) are concentrated in the central area and the low-value spots (red dots) are concentrated in surrounding areas. However, the results of the LISA analysis contains many outliers. The green triangles, indicating high values in low-value areas, mainly found in the surrounding city with higher levels of the elderly population. The red triangle is the low value surrounding by the high-value area, mainly distributed at the boundary of high and low value. Therefore, it is suggested that streets with high levels of RCFs should allocate more resources towards the peripheral when planning, while streets with low levels of RCFs should mainly consider allocating resources to the center of local residential communities. 

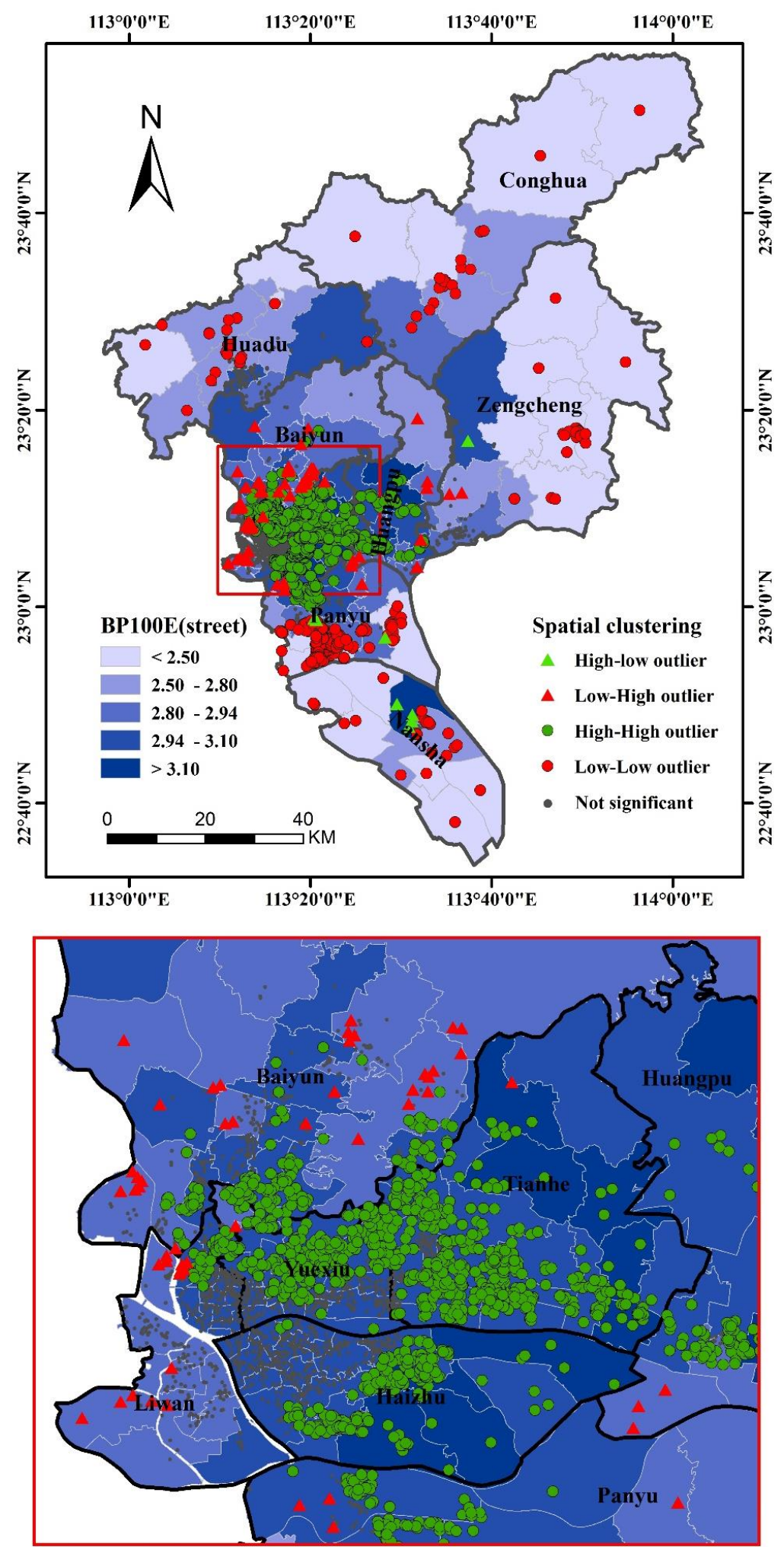

Figure 7. Cluster and outlier analysis using LISA.

\subsection{The Difference of the Accessibilities based on the Two Scales Data}

In order to assess the variation of the accessibility based on street scale, we calculated the maximum difference of accessibility (MDA) to RCFs by two residential communities in the same street. The results are shown in Figure 8. 

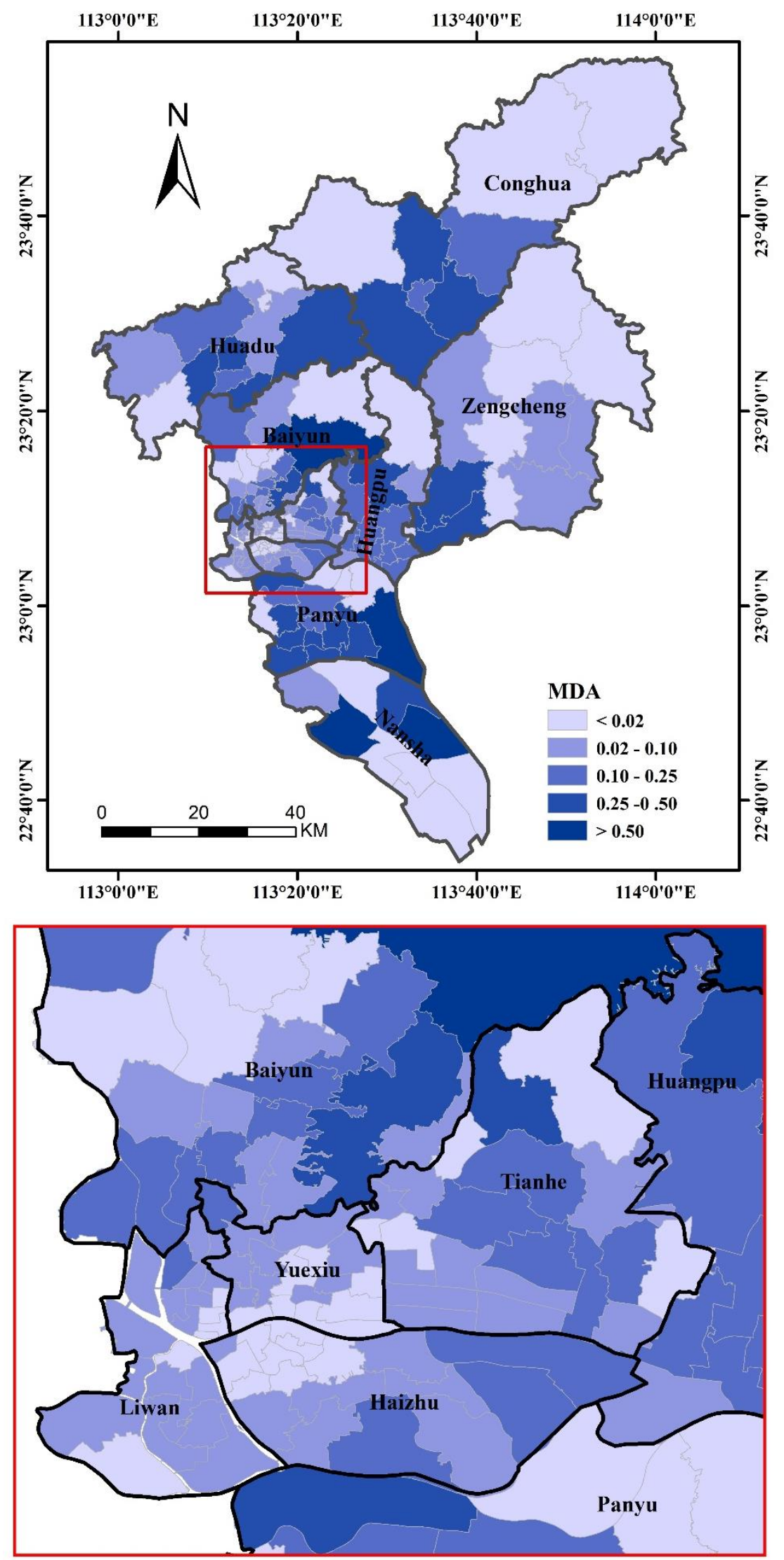

Figure 8. The maximum difference of accessibility (MDA) to RCFs by two residential communities in the same street. 
It was found that the values of MDAs vary considerably among different streets, ranging from less than 0.02 to 0.94 . The difference in accessibility to RCFs shows a significant spatial variation in the streets of Guangzhou. Among those, the differences of accessibilities of residential communities are the largest (MDAs bigger than 0.5) for four streets (towns): Nansha Street, Dagang Town, Shilou Town, and Taihe Town in the main city. However, in the central and surrounding cities, smaller differences are found. Especially in the Yuexiu District of the central city, the value of MDAs is less than 0.1 for most streets (Figure 8).

Regions consisting of centers of main cities demonstrate high differences of accessibilities to RCFs, which roughly form a ring-shaped structure filling the space between the central and surrounding cities in the streets of Guangzhou. Compared to the central and surrounding cities, the elderly person population is medium in the main city and not far away from the central city, which has the maximum RCFs (Table 3). The elderly people having more choices in the main cities lead to large differences in accessibilities. According to the results of MDAs, large errors may occur when assessing the accessibility of a family or residential community based on the street results, especially for the main city.

To further compare and analyze the difference of the accessibilities to the RCFs between the two scales data in Guangzhou, relative and cumulative errors of the accessibilities are calculated in a similar way to the previous studies $[24,25,32]$. Assuming for the $\mathrm{n}$ residential communities in a street, the accessibilities to the RCFs based on the $\mathrm{n}$ residential communities and the street are $x_{i}(i=1,2 \ldots$ $n$ ) and $x_{s}$, respectively. The relative errors for the $\mathrm{n}$ residential communities could be calculated by Equation (3) and the cumulative error in the street by Equation (4).

$$
\begin{gathered}
R E_{i}=\left|\frac{x_{i}-x_{s}}{x_{s}}\right| \times 100 \%, \\
C E=\sum_{i=1}^{i=n} R E_{i}
\end{gathered}
$$

where $x_{i}$ is the accessibility to the RCFs based on the residential community and $x_{s}$ is the accessibility to the RCFs based on the street.

It was found that very low relative errors for most residential communities in the central city, but significantly higher errors in the surrounding city (Figure 9). Due to sufficiently high residential densities in streets of the areas, the accessibilities based on streets have much better agreement with each residential community. Merely a few residential communities have errors over $5 \%$ in the central city. Residential communities with high errors (more than 10\%) are mainly distributed in the middle of Conghua District and southwest of Zengcheng (Yongning, Zhucun, and Shitan Streets) in the surrounding city, and the middle of Huangpu District (Luogang Street), southeast of Panyu District (Shilou Town), and the east of Nansha District (Nansha and Huangge Streets) in the main city. 

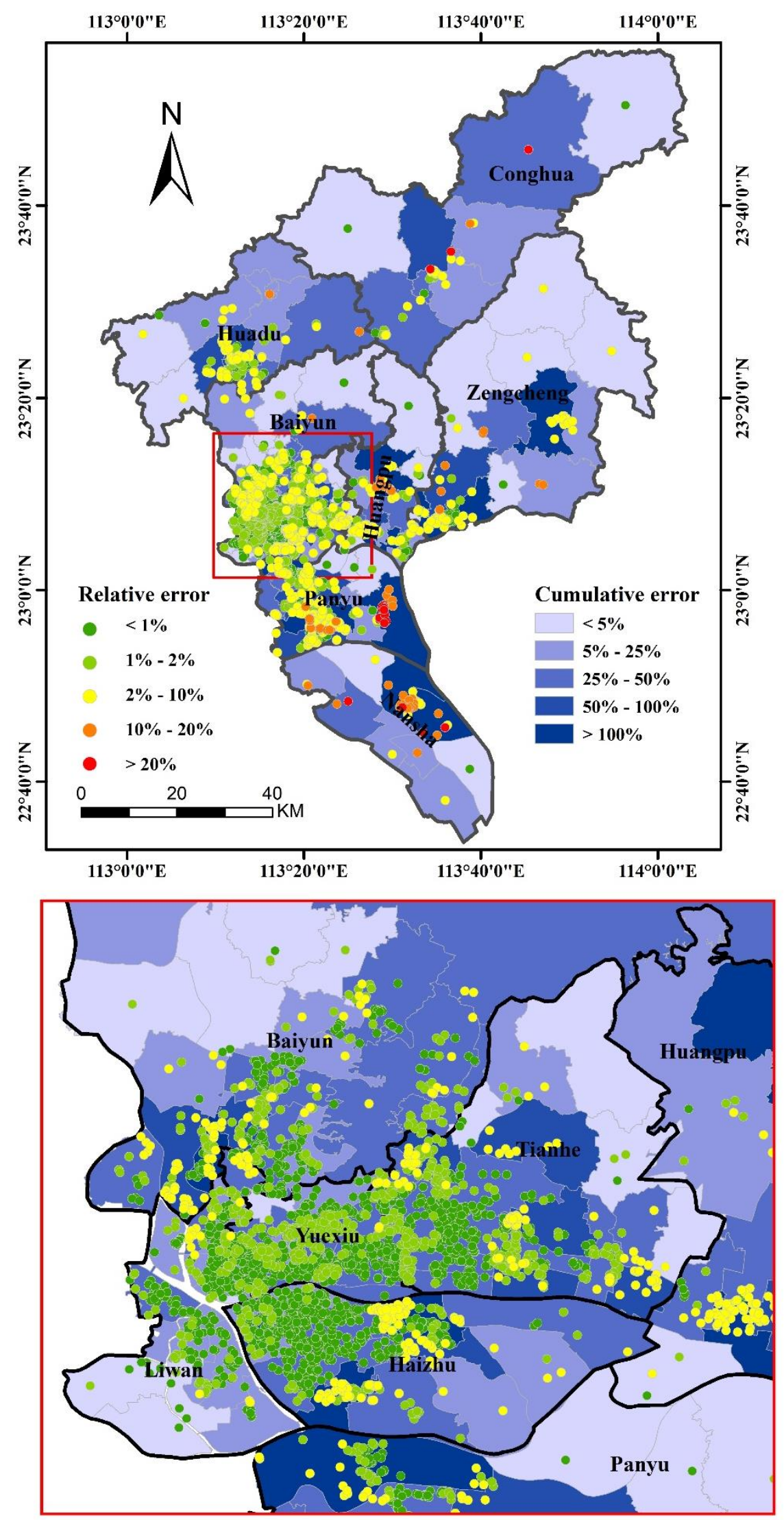

Figure 9. The relative error between the accessibilities to RCFs based on residential community and street scales data, and the cumulative error in a street. 
For the accessibility of each residential community in Guangzhou, the relative error appears to be very low. However, there are more than 20 residential communities in a street (Table 1), where the accumulative effects are large (Figure 9). It was found that the cumulative error increases rapidly in the streets, over $25 \%$ in major streets of the central city. Among them, the cumulative errors of Chigang and Ruibao Streets increased to even more than $100 \%$ in Haizhu District. However, in other streets of northern Baiyun, Huangpu and Zengcheng Districts, the cumulative errors increased relatively slowly. However, this is due to the fact that few residential community data are obtained from these streets. From the analysis above, it is evident that the accessibility to RCFs based on the street leads to large cumulative errors to the residential communities.

Finally, we calculate the change of RCFs beds (CB) and the corresponding affected elderly population (AP) by the accessibilities to the RCFs and the total elderly population in the streets of Guangzhou (Figure 10). Based on the accessibilities, the available beds for the elderly in a street can be calculated by Equation (5), so to the residential communities in the street. Then, $\mathrm{CB}$ represents the difference of the sum of available beds based on the residential communities and street (Equation (6)). According to the report of the development plan of the elderly care service in Guangzhou, the goal of accessibility to RCFs should reach four beds per hundred elderly people [4]. Therefore, AP could be calculated based on CB in the street (Equation (7)).

$$
\begin{gathered}
\text { Beds }=\text { Population } \times x \%, \\
C B=\mid B e d s_{\text {street }}-\sum_{i}^{n} \text { Beds }_{\text {residential communities }} \mid \\
A P=C B \div 4 \times 100
\end{gathered}
$$

where $x$ is the accessibility to the RCFs, Beds street and Beds residential communities $_{\text {are the available beds for }}$ the elderly in a street and a residential community, respectively.

In the central city, it was found that the differences of the accessibilities based on the two- scales data (streets and its internal residential communities) are less than 10 beds in most streets. However, towards the main city, the difference increases gradually. For example, the differences in Shilou Town, Panyu District, and Dagang Town, Nansha District are over 80 beds (Figure 10). For the corresponding affected elderly population, it was found that almost $90 \%$ of streets would affect more than 50 elderly persons (Fig. 10). In addition, there were 14 streets with over 500 elderly persons were affected. Among them, seven streets are in the main city and others in the surrounding city. Due to the large elderly population and the significant variation of the accessibilities of residential communities, there are six streets have more than 1000 elderly person affected: Chengjiao Street in Conghua District, Jianggao Town in Baiyu District, Yongning and Shitan Streets in Zengcheng District, Shilou Town in Panyu District and Dagang Town in Nansha District (Figure 10).

Based on the analysis above, we conclude that the spatial accessibility to RCFs by street (town) scale data ignoring the significant variations of its internal residential communities leads to large cumulative error in Guangzhou, which might have a significant impact on hundreds of the elderly population per street. Measurement and analysis of the spatial accessibility to RCFs based on the smaller-scale residential community data can provide a more precise result and better reference to a family, especially in the area similar to the main city of Guangzhou. 

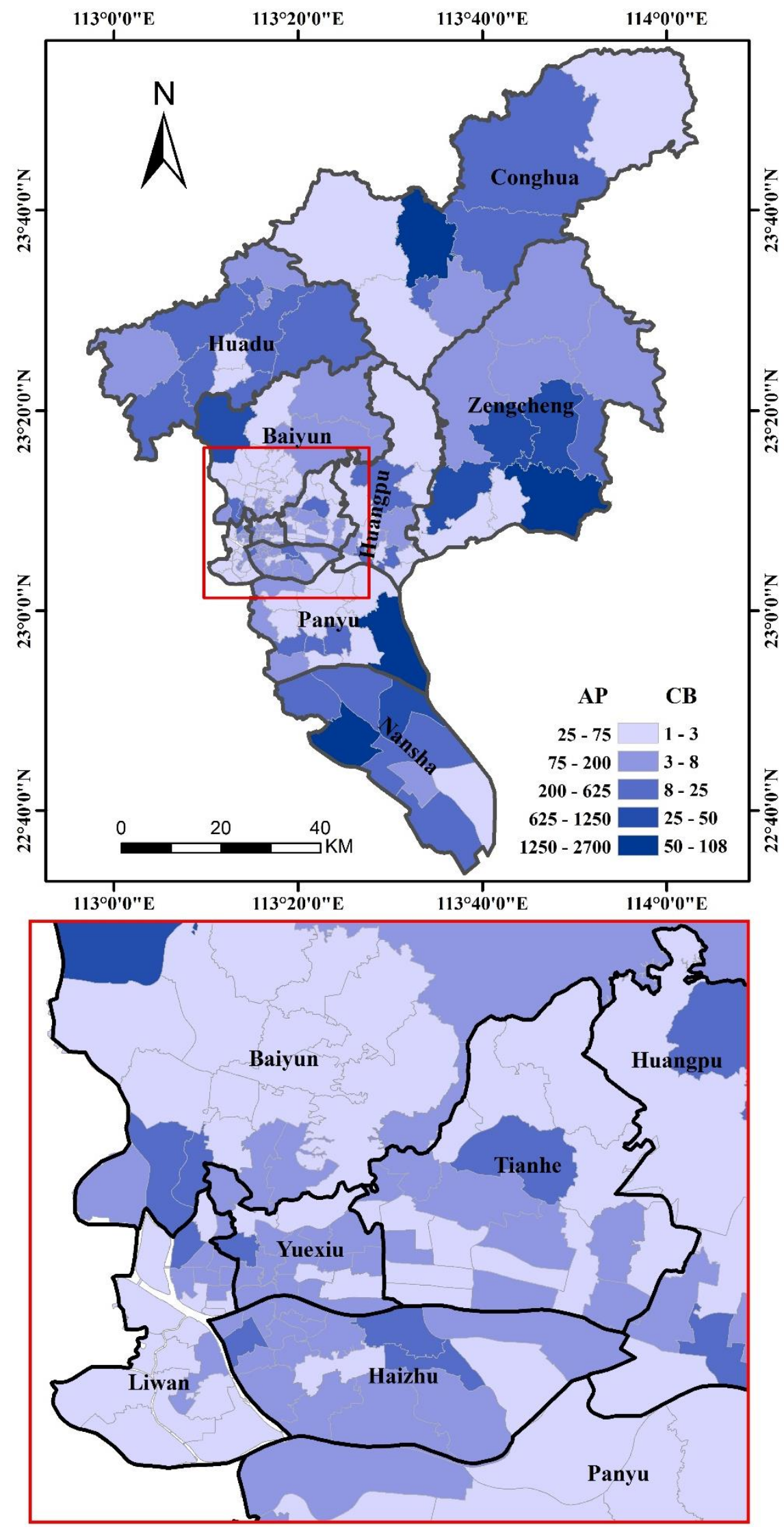

Figure 10. The change of RCFs beds (CB) and the corresponding affected elderly population (AP) in the streets in Guangzhou. 


\section{Conclusions and Suggestions for Future Urban Planning}

Measurement and analysis of the accessibilities to RCFs based on the residential community and street scales data in Guangzhou are presented. It was found that both the elderly and the service-dense blobs of the RCFs show a high-medium-low three-level spatial distribution pattern and characterized by central clustering and peripheral dispersion (Figure 4, Figure 5).

The accessibilities to RCFs mainly range from less than 2.5 beds to 3.45 beds in Guangzhou and the results based on the residential community scale are generally consistent with those findings from the street scale: the closer to the central area, the higher the accessibility (Figure 6). Besides, the accessibility shows a certain degree of spatial clustering and heterogeneity (Figure 7).

There existed a big difference between the results based on the two scales of data. It was found that the maximum difference in the accessibility of two residential communities in the same street ranged from less than 0.02 to 0.94 in Guangzhou (Figure 8). Places with higher values consisting of mainly of centers from main cities form a ring-shaped region between the central and surrounding cities. Although the relative errors of the accessibility results based on the two scales data are relatively low, cumulative errors can be large, with over $25 \%$ in major streets of the central and main cities. In 14 streets of Haizhu, Baiyun, Huangpu Panyu, Zengcheng and Nansha Districts, the cumulative errors of increased even exceed 100\% (Figure 9).

The differences and errors affect a large number of the elderly population. Almost $90 \%$ of streets in Guangzhou has more than 50 elderly persons affected, with six over than 1000 (Figure 10).

In the central city, despite the abundance of RCFs and expedite traffic, it will be still hard to meet the requirements of society aging, due to the sheer size of the elderly population and the lack of available land. Therefore, a potential solution is to maximize RCFs in the edge area of the central city or in the main cities well connected to the central city.

Due to low density, a large number of RCFs and expedite traffic, the accessibilities of Baiyun, Huangpu and Panyu Districts are generally high in the main city. On the one hand, we recommend expanding the scale of existing facilities to meet the needs of the local and the urban residents who migrate from the central city. On the other hand, developing high-quality senior citizen service institutions would meet the needs on different levels. Nansha District has a large area but inconvenient transportation. Therefore, its accessibility is low. It is recommended to fully exploit the potential of existing RCFs and improve their service capability by restructuring and expansion.

In the surrounding cities, we see decreasing accessibility with distance from the main city. In general, low accessibility is mainly caused by the sparsity of RCFs, uneven distribution, and poor traffic. Thus, we recommend building new RCFs in the area where RCFs are scarce. The development of vacation-oriented resources may also be promising for elderly people in areas with suitable environments and convenient transportation. In addition, private care institutions should be encouraged to promote the layout of senior care service institutions facilitating more rational planning to the spatial distribution of RCFs in Guangzhou and other aging cities.

Author Contributions: Conceptualization, D.W. and C.W.; Methodology, C.Q. and S.L.; Formal analysis, C.Q.; Investigation, D.W. and P.H.; Data Curation, S.L. and P.H.; Writing-Original Draft Preparation, D.W. and C.Q.; Writing-Review \& Editing, C.W. and D.W.; Supervision, J.Y., Y.L. and P.H.; Project Administration, S.L. Funding Acquisition, D.W., C.W. and C.Q. All authors have read and agreed to the published version of the manuscript.

Funding: This research was supported by National Natural Science Foundation of China (41801364), the research on spatial distribution of Residential Care Facilities in Guangzhou (2017YB002), Key Special Project for Introduced Talents Team of Southern Marine Science and Engineering Guangdong Laboratory (Guangzhou) (GML2019ZD0301), Key Scientific Research Projects of Henan Colleges and Universities (19A420001), GDAS' Project of Science and Technology Development (2020GDASYL-20200302001, 2018GDASCX-0905, 2020GDASYL-20200104006 and 2019GDASYL-0301001).

Acknowledgments: Authors appreciate the constructive suggestions from reviewers and editors that helped improve this paper.

Conflicts of Interest: The authors declare no conflict of interest. 


\section{References}

1. Zhou, R.; Zhuang, R.; Huang, C. Pattern evolution and formative mechanism of aging in China. Acta Geogr. Sin. 2019, 74, 2163-2177.

2. Cheng, Y.; Rosenberg, M.; Wang, W.; Yang, L.; Li, H. Aging, health and place in residential care facilities in Beijing, China. Soc. Sci. Med. 2011, 72,365-372. [CrossRef] [PubMed]

3. Cheng, Y.; Wang, J.E.; Rosenberg, M. Spatial access to residential care resources in Beijing, China. Int. J. Health Geogr. 2012, 11, 32-42. [CrossRef] [PubMed]

4. Available online: http://www.gz.gov.cn/zfjgzy/gzswsjkwyh/zdlyxxgk/jbylws/ggws/content/post_2996619. html (accessed on 14 April 2020).

5. Available online: http://mzj.gz.gov.cn/gkmlpt/content/4/4460/post_4460876.html (accessed on 14 April 2020).

6. Billaudeau, N.; Oppert, J.; Simon, C.; Charreire, H.; Casey, R.; Salze, P.; Badariotti, D.; Banos, A.; Weber, C.; Chaix, B. Investigating disparities in spatial accessibility to and characteristics of sportfacilities: Direction, strength, and spatial scale of associations with area income. Health Place 2011, 17, 114-121. [CrossRef]

7. Higgs, G.; Langford, M.; Norman, P. Accessibility to sport facilities in Wales: A GIS-based analysis of socioeconomic variations in provision. Geoforum 2015, 62, 105-120. [CrossRef]

8. Tao, Z.; Cheng, Y.; Dai, T.; Li, X. Spatial optimization of residential care facility locations in 2020 in Beijing: Maximum equity in accessibility. Prog. Geogr. 2015, 34, 1609-1616.

9. Dennis, K.; Luis, R.; Erick, G.; Rebecca, L.; Patrick, S. Spatial accessibility of drug treatment facilities and the effects on locus of control, drug use, and service use among heroin-injecting Mexican American men. Int. J. Drug Policy 2014, 25, 598-607.

10. Gharani, P.; Stewart, K.; Ryan, G. An enhanced approach for modeling spatial accessibility for In Vitro fertilization services in the rural Midwestern United States. Appl. Geogr. 2015, 64, 12-23. [CrossRef]

11. Kayleigh, B.; James, A.; Jacqueline, M.; Kloppd, J. Accessibility across transport modes and residential developments in Nairobi. J. Transp. Geogr. 2019, 74, 77-90.

12. Bauer, J.; Müller, R.; Brüggmann, D.; Groneberg, D.A. Spatial accessibility of primary care in England: A cross-sectional study using a floating catchment area method. Health Serv. Res. 2018, 53, 1957-1978. [CrossRef]

13. Chen, X.; Jia, P. A comparative analysis of accessibility measures by the two-step floating catchment area (2SFCA) method. Int. J. Geogr. Inf. Sci. 2019, 33, 1739-1758. [CrossRef]

14. Guagliardo, M.F. Spatial accessibility of primary care concepts, methods and challenges. Int. J. Health Geogr. 2004, 3, 3-15. [CrossRef] [PubMed]

15. Radke, J.; Mu, L. Spatial decompositions, modeling and mapping service regions to predict access to social programs. Geogr. Inf. Sci. 2000, 6, 105-112. [CrossRef]

16. Stentzel, U.; Bahr, J.; Fredrich, D.; Piegsa, J.; Hoffmann, W.; Berg, N. Is there an association between spatial accessibility of outpatient care and utilization? Analysis of gynecological and general care. BMC Health Serv. Res. 2018, 18, 322. [CrossRef]

17. Tao, Z.; Yao, Z.; Kong, H.; Duan, F.; Li, G. Spatial accessibility to healthcare services in Shenzhen, China: Improving the multimodal two-step floating catchment area method by estimating travel time via online map APIs. BMC Health Serv. Res. 2018, 18, 345. [CrossRef]

18. Yin, C.; He, Q.; Liu, Y.; Chen, W.; Gao, Y. Inequality of public health and its role in spatial accessibility to medical facilities in China. Appl. Geogr. 2018, 92, 50-62. [CrossRef]

19. Yu, W.; Ai, T.; Li, J.; Yang, M.; Shuai, Y. Potential change of spatial accessibility to health services with the opening of private streets in Shenzhen, China. IEEE Access 2018, 6, 72824-72835. [CrossRef]

20. Tao, Z.; Cheng, Y.; Dai, T. Measuring spatial accessibility to residential care facilities in Beijing. Prog. Geogr. 2014, 33, 616-624.

21. Yang, D.; Goerge, R.; Mullner, R. Comparing GIS-based methods of measuring spatial accessibility to health services. J. Med. Syst. 2006, 30, 23-32. [CrossRef]

22. KC, K.; Corcoran, J.; Chhetri, P. Measuring the spatial accessibility to fire stations using enhanced floating catchment method. Socio Econ. Plan. Sci. 2020, 69, 100673. [CrossRef]

23. Tao, Z.; Cheng, Y. Research progress of the two-step floating catchment area method and extensions. Prog. Geogr. 2016, 35, 589-599. 
24. McGrail, M.R.; Humphreys, J.S. Measuring spatial accessibility to primary care in rural areas: Improving the effectiveness of the two-step floating catchment area method. Appl. Geogr. 2009, 29, 533-541. [CrossRef]

25. Ouko, J.; Gachari, M.; Sichangi, A.; Alegana, V. Geographic information system-based evaluation of spatial accessibility to maternal health facilities in Siaya County, Kenya. Geogr. Res. 2019, 57, 286-298. [CrossRef]

26. Jia, P.; Shi, X.; Xierali, I. Teaming up census and patient data to delineate fine-scale hospital service areas and identify geographic disparities in hospital accessibility. Env. Monit Assess 2019, 191, 303. [CrossRef] [PubMed]

27. Song, Z.; Chen, W.; Zhang, G.; Zhang, L. Spatial accessibility to public service facilities and its measurement approaches. Prog. Geogr. 2010, 29, 1217-1224.

28. Wang, F. Quantitative Methods Applications in GIS; The Commercial Press: Beijing, China, 2011.

29. Available online: http://www.stats.gov.cn/tjsj/pcsj/rkpc/6rp/indexch.htm (accessed on 14 April 2020).

30. Luo, W.; Wang, F.H. Measures of spatial accessibility to health care in a GIS environment: Synthesis and a case study in the Chicago region. Environ. Plan. B Plan. Des. 2003, 30, 865-884. [CrossRef]

31. Wang, D.; Bao, S.; Wang, C.; Wang, C. Agricultural meteorological disaster grading in Guangdong Province based on data mining. J. Disaster Res. 2016, 12, 187-197. [CrossRef]

32. Xie, Y.; Sha, Z.; Yu, M.; Bai, Y.; Zhang, L. A comparison of two models with Landsat data for estimating above ground grassland biomass in Inner Mongolia, China. Ecol. Model. 2009, 220, 1810-1818. [CrossRef]

(C) 2020 by the authors. Licensee MDPI, Basel, Switzerland. This article is an open access article distributed under the terms and conditions of the Creative Commons Attribution (CC BY) license (http://creativecommons.org/licenses/by/4.0/). 